\title{
A Case Study of Process Facility Optimization using Discrete Event Simulation and Genetic Algorithm
}

\author{
K.P. Dahal \\ University of Bradford \\ Bradford, BD7 1DP, UK. \\ Tel: +441274234019 \\ k.p.dahal@bradford.ac.uk
}

\author{
S.J. Galloway, G.M. Burt, J.R. McDonald \\ University of Strathclyde \\ Glasgow, G1 1XW, UK. \\ Tel: +441415484840 \\ \{stuart.galloway, g.burt; j.mcdonald\}@ starth.ac.uk
}

\author{
I. Hopkins \\ Rolls Royce plc. PO Box 31 \\ Derby, DE24 8BJ, UK. \\ Tel: +441332246483 \\ ian.hopkins@rolls-royce.com
}

\begin{abstract}
Optimization problems such as resource allocation, job-shop scheduling, equipment utilization and process scheduling occur in a broad range of processing industries. This paper presents modeling, simulation and optimization of a port facility such that effective operational management is obtained. A GA base approach has been integrated with the port system model to optimize its operation. A case study of bulk material port handling systems is considered.
\end{abstract}

\section{Categories and Subject Descriptors I.6 [Simulation and Modeling]: Discrete event}

General Terms: Algorithms, Management, Design

Keywords: Bulk handling port system, Simulation, Optimization, GA.

\section{INTRODUCTION}

Optimization problems such as resource allocation, job-shop scheduling, equipment utilization, and process scheduling occur in a broad range of industries. Solving these scheduling problems is important for the economic operation of facilities and has been studied in the literature [1-3]. For many real world resource management problems the material flow process yields to the application of many traditional solution techniques [1,2]. Other authors have coupled simulation models with optimization components in an attempt to improve solution quality [3]. In this paper a case study of the effective management of bulk material port processing systems is discussed. A GA with heuristics has been coupled to a discrete event port simulation tool to optimize the port design and operation. The discussion about modelling is limited in this short paper, a fuller description is given in [4].

\section{PORT SYSTEM MODELLING}

During port operation various materials, such as coal, coke, iron ore, iron pellets, are imported to or exported from the bulk facility. Following the arrival of a vessel carrying materials, it is placed on a queue until an appropriate berth and units for the

Copyright is held by the author/owner(s).

GECCO'05, June 25-29, 2005, Washington, DC, USA.

ACM 1-59593-010-8/05/0006. berth become available. Unloaders are used to unload material from the vessel. The unloaders transfer the material to an importing conveyor system. The material is either collected from a conveyor by a stacker and stored in a stockpile, or fed to a direct demand. Materials not supplying a direct demand are likewise taken away by exporting vessels. Loaders are used to load material to vessels. These components take material from an exporting conveyor, which in turn obtains it from a stockpile through a reclaimer.

The dynamics of port systems, uncertainty in the arrival pattern of the vessels coupled with the random failures of port components makes the mathematical modelling of such facilities difficult. For this reason a discrete event port simulation tool was developed which permits the construction of specific port models from generic component models. The component models are designed for a limited number of contexts but with the flexibility to work in multiple port models. Each port object characterises both the healthy and failed behaviour of equipment of that type. The port simulator facilitates the construction of a simulation model by dragging, dropping, networking and parameterizing the port component models. The simulation of port system operation produces a diverse array of performance related results. Through these performance indices various forms of assessment both economic and operational can be determined.

\section{GA OPTIMIZER}

The port simulator as designed does not inherently provide any optimization capability. Therefore, a GA based optimizer has been developed separately and integrated with the port simulator as shown in Figure 1. Once a port simulation model is constructed all the controlling factors, physical variables (such as, sizing of components) and decision variables (such as, queuing strategies) in the port system are presented for user selection. These user defined variables are used to form GA chromosome, which retains a fixed length through out the duration of the optimization. The range for each of the different variables is predefined by a suitable mapping from the physical variables of the problem.

The goodness (evaluation value) of a trial solution is calculated by using an evaluation function, which is constructed using port system performance measures. The user selected performance measuring criteria and penalty factors make up the evaluation function. The evaluation function is the weighted sum of the penalty values for each constraint violation and the normalized objective function itself. 


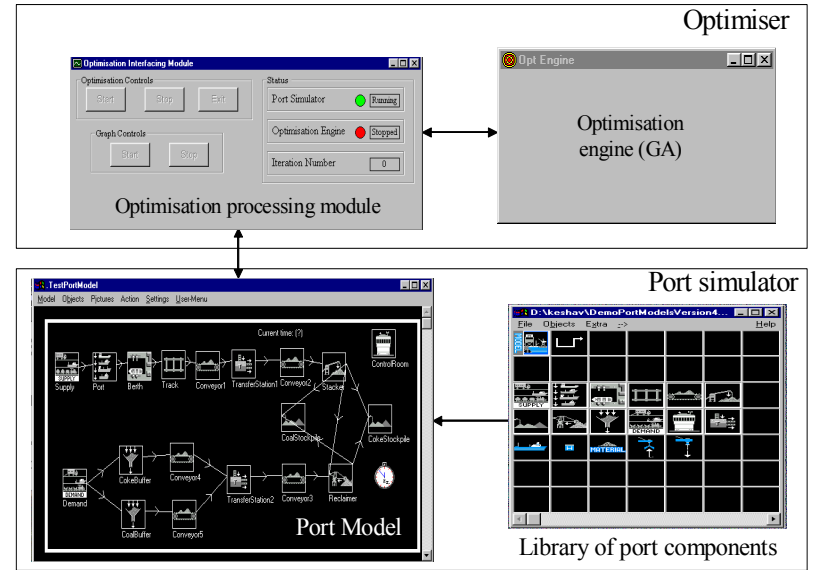

Figure 1. Port simulation and optimization software.

The GA performance is generally dependent on the particular GA structure, operators and parameters used. A range of GA operators/parameters are provided for user selection. The appropriate operators/parameters are decided after experimentation. The stopping criterion used is either a maximum iteration number or a specified number of iterations for which there is no improvement in the solution quality.

\section{CASE STUDY}

A simple case study of port optimization, which is based on the operation of a real-world port facility, is considered here to demonstrate the application of the tool. The port system includes both supply and demand sides (see port model in Figure 1). The supply side consists of two unloaders, two conveyors and a stacker. Two types of vessels bringing two different material types arrive at the port model on a regular basis - with a degree of uncertainty. The materials must be stored in separate stockpiles. The demand side of the port model consists of a reclaimer and three conveyors. The out-going materials are being used to feed two separate demands, one for each material type.

The optimization problem considered here is to determine the best operational strategies and equipment sizes for the given configuration of the facility, the vessel arrival patterns, the contents of ships, and the plant capacity limitations are given. We want to minimize total facility operating costs by reducing delays to vessels, maximizing utilization of equipment, ensuring continuous and steady supply of materials as demanded by subsequent facilities over a simulation period. The weighted sum of the normalized values of these objective function and the penalty values for each constraint violation gives the evaluation measure of a trial solution. A single simulation of the port model was performed. One way to improve the results obtained from the port system model is to execute a number of different scenarios. This requires by redefining the required parameters in the model and repeating the simulation. However, often such a trial and error approach can result in an unfocused search through the possible alternatives and consequently, the solution that yields the best improvement is often missed. Through the use of the optimization approach a more focused and unbiased method can be used to determine a good solution for the port system.

The GA optimizer has been applied for this case study using the steady state population updating approach, ranking selection operator, two point crossover, random mutation operator and the elitist approach. Ten GA runs have performed with different initial populations. Parts of these initial population pool have been generated either using heuristics or randomly to provide diverse solutions. In the initial stage the GA explored diverse areas of the search space. The good solutions are located towards the end of the iterative process where the improvement exhibited in the solution quality due to a consequence of the genetic operators.

The port specification (the sizing and decision parameters) and performance measures identified by the GA run which gave in the best evaluation measure shows that the GA solution has reduced the total demurrage costs by $38 \%$ at the expense of a $1 \%$ reduction in utilization with respect to the simulation solution, while there is no significant change in the average stockpile levels for both the stockpiles.

\section{CONCLUSIONS}

A GA-based approach with port heuristics and operational rules has been integrated with a discrete event simulation model to solve optimization problems that occur in the port processing facility. A generic port modeling tool was developed that characterizes the port components and their complex interaction, and therefore a complex mathematical model need not be developed for optimization purposes. Instead, the simulation model is used as part of the GA evaluation function with repairing and filtering of the trial solutions. A case study based on real world port systems was presented and a significant improvement is demonstrated in the operational and economic performance as a result of the GA and simulation model interaction.

\section{REFERENCES}

[1] Shah, N., Mathematical Programming Techniques for Crude Oil Scheduling, Computers Chem. Eng. 20, 1227-1232, 1996.

[2] Sasikumar, M., et al, PIPES: A Heuristic Search Model for Pipeline Schedule Generation. Knowledge-Based Systems 10, 169-175, 1997.

[3] Hart, R. P. S., et al., Optimization Techniques for a Computer Simulation of a Pastoral Dairy Farm, Computers Electronics in Agriculture 19, 129-153, 1998.

[4] Dahal K.P., Galloway S.J., Burt G.M., McDonald J.R. and Hopkins J.R., A port system simulation facility with an optimisation capability, International Journal of Computational Intelligence and Applications (IJCIA), 3 (4), 395-410, 2003. 